\title{
First combined search for neutrino point-sources in the Southern Sky with the ANTARES and IceCube neutrino telescopes
}

\author{
J. Barrios-Martí \\ Instituto de Física Corpuscular, IFIC (UV-CSIC), Parque Científico, C/Catedrático José Beltrán \\ 2, E-46980 Paterna, Spain \\ E-mail: javier.barrioseific.uv.es \\ Chad Finley \\ Oskar Klein Centre and Dept. of Physics, Stockholm University, SE-10691 Stockholm, Sweden \\ E-mail: cfinleyefysik.su.se
}

on behalf of the ANTARES and IceCube collaborations

\begin{abstract}
A search for cosmic neutrino point-like sources using the ANTARES and IceCube neutrino telescopes over the Southern Hemisphere is presented. The ANTARES data was collected between January 2007 and December 2012, whereas the IceCube data ranges from April 2008 to May 2011. Clusters of muon neutrinos over the diffusely distributed background have been looked for by means of an unbinned maximum likelihood maximisation. This method is used to search for a localised excess of events over the whole Southern Sky assuming an $E^{-2}$ source spectrum. A search over a pre-selected list of candidate sources has also been carried out for different source assumptions: spectral indices of 2.0 and 2.5, and energy cutoffs of $1 \mathrm{PeV}, 300 \mathrm{TeV}$ and $100 \mathrm{TeV}$. No significant excess over the expected background has been found, and upper limits for the candidate sources are presented compared to the individual experiments.
\end{abstract}

The 34th International Cosmic Ray Conference,

30 July- 6 August, 2015

The Hague, The Netherlands

\footnotetext{
*Speaker.
} 


\section{Introduction}

Neutrinos offer unique insight into the Universe due to the fact that they interact only weakly. This also implies that their detection is challenging. The field is presently led by the IceCube [1] and ANTARES [2] experiments. IceCube is the first detector to reach the cubic-kilometer size predicted to be necessary to detect cosmic neutrino fluxes. Recently, IceCube has reported the crucial discovery of a flux of neutrinos up to $\sim \mathrm{PeV}$ energies which cannot be explained by the background of atmospheric muons and neutrinos [3, 4]. Meanwhile the ANTARES experiment has proven the feasibility of the Cherenkov telescope technique in sea water $[5,6]$. While its instrumented volume is significantly smaller than that of IceCube, its geographical location provides a better view of the Southern sky for neutrino energies below $100 \mathrm{TeV}$. This provides better sensitivity to the many predicted Galactic sources of neutrinos in this part of the sky. The complementarity of the detectors for Southern sky sources allows for a gain in sensitivity by combining the analysis of data from both experiments in a joint search for point sources. The improvement with this combination depends on the actual details of the fluxes, in particular the energy spectrum and a possible energy cut-off of the signal. The energy spectra are not yet known and predictions vary widely depending on the source model.

\section{Neutrino Data Samples}

The data sample corresponds to all events from the Southern sky which were included in the three-year IceCube point-source analysis [7] combined with the events in the latest ANTARES point-source analysis [8]. The ANTARES sample corresponds to data recorded from 2007 January 29 to 2012 December 31 . The total number of events in this sample amounts to 5516, of which 4136 are from the Southern Hemisphere. The estimated contamination of mis-reconstructed atmospheric muons is of 10\%. The IceCube data was recorded from 2008 April 5 to 2011 May 13, with a total number of 146018 events in the Southern Sky. In contrast to the ANTARES sample, these events are predominantly atmospheric muons rather than atmospheric neutrinos, because the Earth cannot be used as a neutrino filter for directions above the detector.

The fraction of expected source events needs to be calculated in order to estimate the relative contribution of each sample in the likelihood. This quantity is defined as the ratio of the expected number of signal events from the given sample to the expected number from all samples,

$$
C^{j}\left(\delta, d \Phi / d E_{v}\right)=\frac{N^{j}\left(\delta, d \Phi / d E_{v}\right)}{\sum_{i} N^{i}\left(\delta, d \Phi / d E_{v}\right)},
$$

where the total number of expected events for the $j$-th sample, $N^{j}$, with a given source declination, $\delta$, and a given source spectrum, $\frac{d \Phi}{d E_{v}}$, can be calculated as

$$
N^{j}\left(\delta, \frac{d \Phi}{d E_{v}}\right)=\int d t \int d E_{v} A_{\mathrm{eff}}^{j}\left(E_{v}, \delta\right) \frac{d \Phi}{d E_{v}} .
$$

The time integration extends over the live time of each sample and $A_{\mathrm{eff}}^{j}\left(E_{v}, \delta\right)$ indicates the effective area of the corresponding detector layout $j$ as a function of the neutrino energy, $E_{v}$, and the declination of the source, $\delta$. The declination of a given event is not directly related with the 
zenith direction in the ANTARES telescope, and therefore, the effective area for a given declination changes at different times of the day. Steady, non time-dependent sources are assumed for this analysis. Therefore, it is possible to integrate the zenith dependence for the considered period.

Since each detector layout has a different response depending on the neutrino energy and declination, this relative fraction of source events needs to be calculated for different source spectra and source declinations. Figure 1 shows the relative fraction of signal events for an unbroken $E^{-2}$ spectrum, which corresponds to the standard first order Fermi spectrum [9, 10]. In this case, there is a significant contribution from all samples over most of the Southern Sky, with the ANTARES contribution being more significant for declinations closer to $\delta=-90^{\circ}$, and IceCube for declinations closer to $0^{\circ}$.

Other source assumptions are also considered in this analysis. The relative fraction of source events is also calculated for an unbroken $E^{-2.5}$ power-law spectrum, as suggested in recent IceCube diffuse-flux searches [11], and for an $E^{-2}$ spectrum with an exponential square-root cut-off $\left(\frac{d \Phi}{d E} \propto\right.$ $E^{-2} \exp \left[-\sqrt{\frac{E}{E_{\text {cut-off }}}}\right]$ ) for energy cut-offs of $100 \mathrm{TeV}, 300 \mathrm{TeV}$ and $1 \mathrm{PeV}$, since a square-root dependence may be expected from Galactic sources [12]. Figure 2 shows the relative fraction of source events for these cases. Compared with an unbroken $E^{-2}$ spectrum, the contribution of high energy neutrinos in all of these cases is lower, and therefore the relative contribution of the ANTARES sample increases.

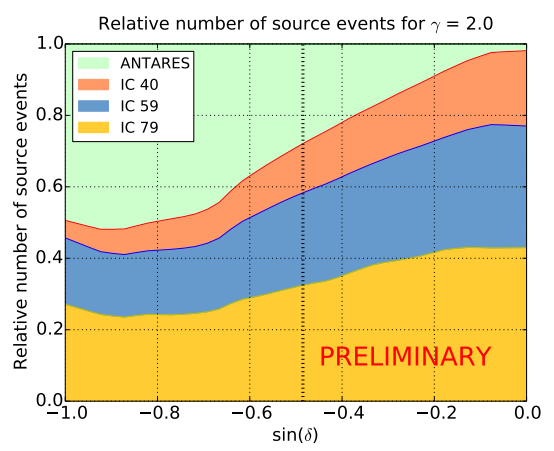

Figure 1: Relative fraction of signal events for each sample as a function of the source declination for the case of an $E^{-2}$ energy spectrum. The orange, blue, and yellow shaded areas correspond respectively to the IceCube 40, 59 and 79-string data samples, and the green shaded area indicates the ANTARES sample. The relative fraction of signal events is used as part of the likelihood function calculation during the search.

\section{Search method}

An unbinned maximum likelihood ratio estimation has been performed to search for excesses of events that could indicate cosmic neutrinos coming from a source. In order to estimate the significance of a cluster of events, this likelihood takes into account the energy and directional information of each event. The data sample to which an event belongs is also taken into account, due to the differences in detector response. The likelihood, as a function of the total number of fitted signal events, $n_{s}$, can be expressed as

$$
L\left(n_{s}\right)=\prod_{j=1}^{4} \prod_{i=1}^{N^{j}}\left[\frac{n_{s}^{j}}{N^{j}} S_{i}^{j}+\left(1-\frac{n_{s}^{j}}{N^{j}}\right) B_{i}^{j}\right],
$$



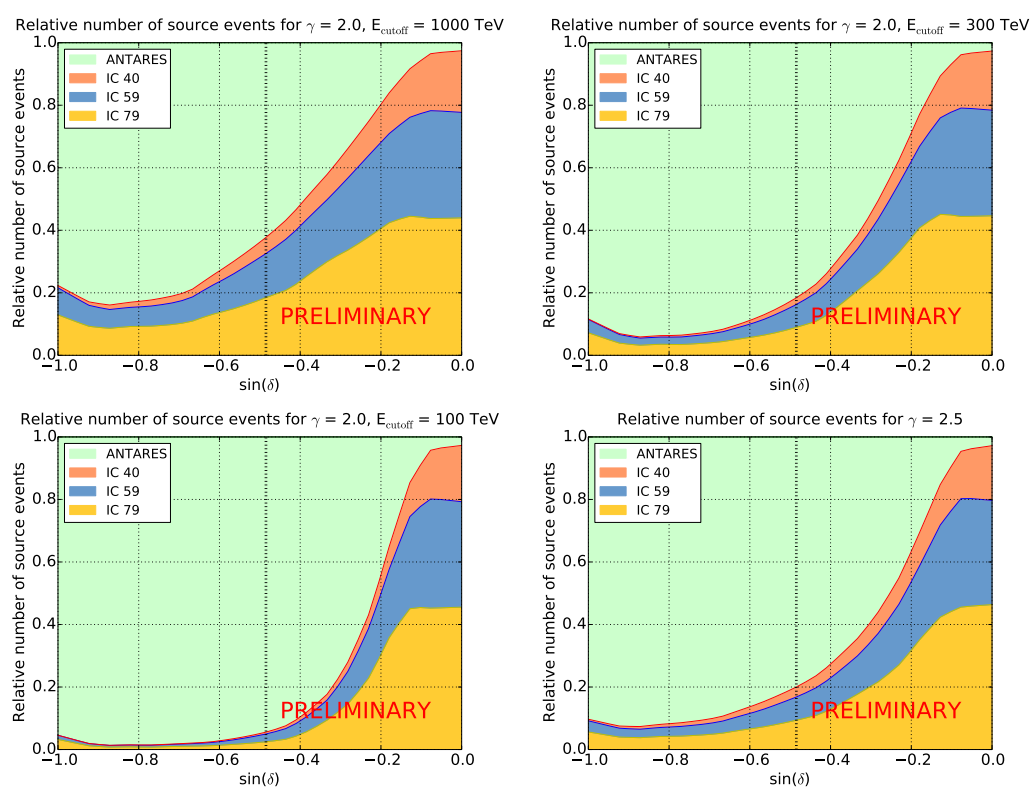

Figure 2: Relative fraction of signal events of each sample as a function of the source declination for different energy spectra: $E^{-2}$ with energy cutoff $E_{\text {cutoff }}$ of $1 \mathrm{PeV}$ (top-left), $300 \mathrm{TeV}$ (top-right), $100 \mathrm{TeV}$ (bottom-left); and $E^{-2.5}$ spectrum (bottom-right). The orange, blue and yellow shaded areas correspond to the IceCube 40, 59 and 79-string data samples, respectively, and the green shaded area indicates the ANTARES sample. The relative fraction is used as part of the likelihood function calculation during the search.

where $j$ indicates one of the four data samples (ANTARES, IC40, IC59 or IC79), $i$ indicates an event belonging to the $j$-th sample, $S_{i}^{j}$ is the value of the signal probability distribution function (PDF) for the $i$-th event in the $j$-th sample, $\mathrm{B}_{i}^{j}$ indicates the value of the background PDF, $N^{j}$ is the total number of events in the $j$-th sample, and $n_{s}^{j}$ is the number of signal events fitted for in the $j$-th sample. Since a given evaluation of the likelihood refers to a single source hypothesis at a fixed sky location, the number of signal events $n_{s}^{j}$ that is fitted for in each sample is related to the total number of signal events $n_{s}$ by the relative contribution of each sample, $n_{s}^{j}=n_{s} \cdot C^{j}\left(\delta, \frac{d \Phi}{d E}\right)$.

The signal and background PDFs for the IceCube and ANTARES samples have slightly different definitions. The signal PDF for ANTARES is defined as

$$
S^{A N T}=\frac{1}{2 \pi \sigma^{2}} \exp \left(-\frac{\Delta \Psi\left(\vec{x}_{s}\right)^{2}}{2 \sigma^{2}}\right) P_{s}^{A N T}\left(\mathscr{N}^{\text {hits }}, \sigma\right),
$$

where $\vec{x}_{s}=\left(\alpha_{s}, \delta_{s}\right)$ indicates the source direction in equatorial coordinates, $\Delta \Psi\left(\vec{x}_{s}\right)$ is the angular distance of a given event to the source, $\sigma$ is the angular error estimate, and $\mathrm{P}_{s}^{A N T}\left(\mathscr{N}^{\text {hits }}, \sigma\right)$ is the probability for a signal event to be reconstructed with an angular error estimate of $\sigma$ and a number of hits taken in the event reconstruction $\mathscr{N}^{\text {hits }}$. The number of hits is a proxy for the energy of the event [13].

The definition of the signal PDFs for the IceCube samples is similar,

$$
S^{I C}=\frac{1}{2 \pi \sigma^{2}} \exp \left(-\frac{\Delta \psi\left(\vec{x}_{s}\right)^{2}}{2 \sigma^{2}}\right) P_{s}^{I C}(\mathscr{E}, \sigma \mid \delta),
$$


where the main difference lies in the use of the reconstructed energy, $\mathscr{E}$, and the declination dependence of the probability for a signal event to be reconstructed with a given $\sigma$ and $\mathscr{E}$. Details about the reconstructed energy proxy can be found in [7] and [14]. The declination dependence is needed mainly because of the event selection cut on reconstructed energy, which is designed to reduce the atmospheric muon background.

Background events are expected to be distributed uniformly in right ascension. The background PDFs are in fact obtained from the experimental data itself. The definitions of the PDFs are:

$$
B^{A N T}=\frac{B^{A N T}(\delta)}{2 \pi} P_{b}^{A N T}\left(\mathscr{N}^{h i t s}, \sigma\right), \quad B^{I C}=\frac{B^{I C}(\delta)}{2 \pi} P_{b}^{I C}(\mathscr{E}, \sigma \mid \delta),
$$

where $\mathrm{B}(\delta)$ is the per-solid-angle rate of observed events as a function of the declination in the corresponding sample. $P_{b}^{A N T}\left(\mathscr{N}^{\text {hits }}, \sigma\right)$ and $P_{b}^{I C}(\mathscr{E}, \sigma \mid \delta)$ characterize the distributions for background event properties, in analogy with the definitions of $P_{s}^{A N T}$ and $P_{s}^{I C}$ for signal events given above.

The test statistic, TS, is determined from the likelihood (Eq. 3.1) as TS $=\log L\left(\hat{n}_{s}\right)-\log L\left(n_{s}=\right.$ 0 ), where $\hat{n}_{s}$ is the value that maximizes the likelihood. The larger the TS, the lower the probability (p-value) of the observation to be produced by the expected background. Simulations are performed to obtain the distributions of the TS. The significance (specifically, the p-value) of an observation is determined by the fraction of TS values which are larger or equal to the observed TS.

The TS is calculated as a preliminary step to obtain the post-trial p-values of a search. TS distributions for the fixed-source, background-only hypotheses have been calculated in steps of $1^{\circ}$ in declination from pseudo-data sets of randomized data. Because these distributions vary with declination, the preliminary TS is turned into a "pre-trial p-value" by comparing the TS obtained at the source location being examined to the background TS distribution for the corresponding declination. Post-trial significance is then estimated with pseudo-data sets and according to the type of search, as explained together with the results in Section 4.

Two different searches for point-like neutrino sources have been performed. In the candidate list search, a possible excess of neutrino events is looked for at the location of 40 pre-selected neutrino source candidates. Since the location of these sources is fixed (at known locations with an uncertainty below the angular resolution of all samples) only the number of signal events $n_{s}$ is a free parameter in the likelihood maximisation. These candidates correspond to all sources in the Southern sky considered in the previous candidate-source list searches performed in the ANTARES and IceCube point-source analyses [8] [7].

The second search is a "full sky" search, looking for a significant point-like excess anywhere in the Southern sky. For this purpose, the likelihood is evaluated in steps of $1^{\circ} \times 1^{\circ}$ over the whole scanned region. Since the angular resolution of both telescopes is smaller than the cell size, the source position is taken as an additional free parameter of the likelihood to fit the best position within the boundaries.

Both the full Southern sky and candidate-list searches have been performed using an $E^{-2}$ source spectrum in the signal PDFs. The main virtue of the energy term in the PDFs is to add power to distinguish signal neutrinos from the softer spectra of atmospheric neutrinos $\left(\sim E^{-3.7}\right)$ and atmospheric muons $\left(\sim E^{-3}\right)$. Limits for the sources in the candidate list have also been calculated for the source spectra mentioned in section 2 . 


\section{Results}

No significant event clusters are found over the expected background. The most significant cluster in the full-Southern sky search is located at equatorial coordinates $\alpha=332.8^{\circ}, \delta=-46.1^{\circ}$, with best-fit $\hat{n}_{s}=7.9$ and pre-trial p-value of $6.0 \times 10^{-7}$. It's found that $24 \%$ of pseudo-data sets have a smaller p-value somewhere in the sky than is found in the real data; the post-trial significance is thus $24 \%$ ( $0.7 \sigma$ in the one-sided sigma convention). The direction of this cluster is consistent with the second most significant cluster in the previous ANTARES point-source analysis (but also less significant).

\begin{tabular}{lccccccccc}
\hline Name & $\delta\left({ }^{\circ}\right)$ & $\alpha\left({ }^{\circ}\right)$ & $n_{s}$ & $p$ & $\phi_{E^{-2}}^{90 C L}$ & $\phi_{E_{c}=1 P e V}^{90 \% C L}$ & $\phi_{E_{c}=300 T e V}^{90 C L}$ & $\phi_{E_{c}=100 T e V}^{90 C L}$ & $\phi_{E^{-2.5}}^{90 C L}$ \\
\hline HESSJ1741-302 & -30.2 & -94.8 & 1.6 & 0.003 & $2.5 \mathrm{E}-08$ & $7.5 \mathrm{E}-06$ & $5.5 \mathrm{E}-08$ & $7.2 \mathrm{E}-08$ & $1.0 \mathrm{E}-07$ \\
3C279 & -5.8 & -166.0 & 1.1 & 0.05 & $3.1 \mathrm{E}-09$ & $1.0 \mathrm{E}-06$ & $6.5 \mathrm{E}-09$ & $9.2 \mathrm{E}-09$ & $6.7 \mathrm{E}-08$ \\
PKS0548-322 & -32.3 & 87.7 & 0.9 & 0.07 & $1.6 \mathrm{E}-08$ & $5.0 \mathrm{E}-06$ & $3.8 \mathrm{E}-08$ & $4.9 \mathrm{E}-08$ & $1.4 \mathrm{E}-08$ \\
ESO139-G12 & -59.9 & -95.6 & 0.8 & 0.07 & $1.8 \mathrm{E}-08$ & $3.9 \mathrm{E}-06$ & $2.9 \mathrm{E}-08$ & $3.7 \mathrm{E}-08$ & $5.1 \mathrm{E}-08$ \\
HESSJ1023-575 & -57.8 & 155.8 & 0.8 & 0.08 & $1.7 \mathrm{E}-08$ & $3.5 \mathrm{E}-06$ & $2.8 \mathrm{E}-08$ & $3.5 \mathrm{E}-08$ & $4.7 \mathrm{E}-08$ \\
RCW86 & -62.5 & -139.3 & 0.2 & 0.11 & $1.4 \mathrm{E}-08$ & $4.4 \mathrm{E}-06$ & $3.6 \mathrm{E}-09$ & $4.0 \mathrm{E}-08$ & $5.7 \mathrm{E}-08$ \\
\hline
\end{tabular}

Table 1: Pre-trial p-values, $p$, fitted number of source events, $n_{s}$, and $90 \%$ C.L. flux limits, $\Phi_{v}^{90 C L}$ for the different source spectra for the 6 candidate sources with the lowest p-values. Units for the flux limits for the $\mathrm{E}^{-2.5}$ spectra, $\phi_{E^{-2.5}}^{90 \mathrm{C}}$, are given in $\mathrm{GeV}^{1.5} \mathrm{~cm}^{-2} \mathrm{~s}^{-1}$, whereas the rest are in $\mathrm{GeVcm}^{-2} \mathrm{~s}^{-1}$. The sources are sorted by their declination.

The results of the candidate source list search are presented in Table 1. No statistically significant excess is found. The most significant excess for any object in the list corresponds to HESS J1741-302 with a pre-trial p-value of 0.003. To account for trial factors, the search is performed on the same list of sources using pseudo data-sets. $11 \%$ of randomized data sets have a smaller $\mathrm{p}$-value for some source than that found for the real data; the post-trial significance of the source list search is thus $11 \%$ ( $1.2 \sigma$ in the one-sided sigma convention).

Table 1 provides the pre-trial p-values, best-fit signal events $n_{s}$ and flux upper limits (under different assumptions of the energy spectrum) for the six sources with the lowest p-value. Figure 3 shows the sensitivities and limits for this search (assuming an $E^{-2}$ spectrum) in comparison with the previously published ANTARES and IceCube analyses of the same data. The point source sensitivity in a substantial region of the sky, centered approximately at the declination of the Galactic Center $\left(\delta=-30^{\circ}\right)$, can be seen to have improved by up to a factor of two. Similar gains in other regions of the sky can be seen for different energy spectra in Figure 4.

\section{Conclusion}

We have presented the first combined point-source analysis of data from the ANTARES and IceCube detectors. The combination of their different characteristics, in particular IceCube's larger size and ANTARES' location in the Northern hemisphere, complement each other for Southern sky searches. We have calculated the sensitivity to point sources and, with respect to an analysis of either data set alone, found that up to a factor of two improvement is achieved in different regions of the Southern sky, depending on the energy spectrum of the source. Two joint analyses of the data sets have been performed: a search over the whole Southern sky for a point-like excess of neutrino events, and a targeted analysis of 40 pre-selected candidate source objects. The largest excess in 


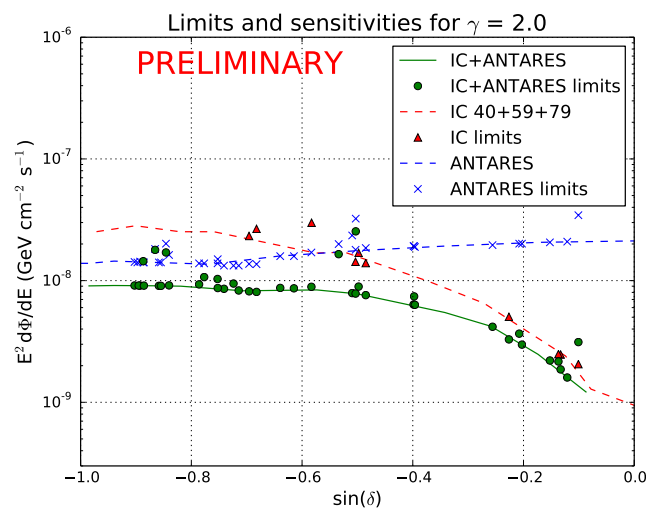

Figure 3: 90\% CL sensitivities and limits (Neyman method) for the neutrino emission from point sources as a function of source declination in the sky, for an assumed $E^{-2}$ energy spectrum of the source. Green points indicate the actual limits on the candidate sources. The green line indicates the sensitivity of the combined search. Blue and red curves/points indicate the published sensitivities/limits for the IceCube and ANTARES analyses, respectively.
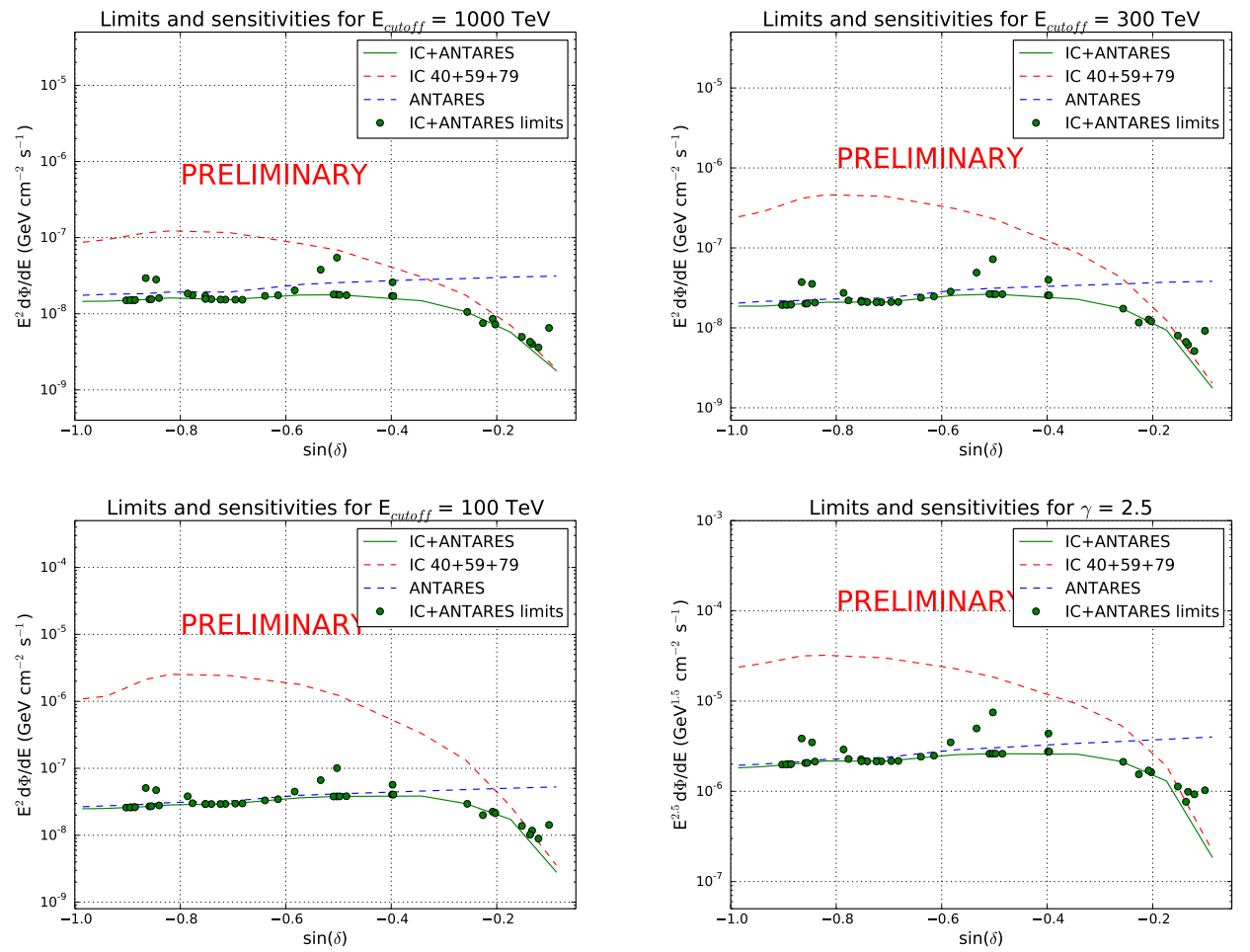

Figure 4: Point source sensitivities and limits for the following energy spectra: $E^{-2}$ with a square-root exponential cut-off at $E=1 \mathrm{PeV}$ (top left), $E=300 \mathrm{TeV}$ (top right), $E=100 \mathrm{TeV}$ (bottom left) and $E^{-2.5}$ unbroken power-law (bottom right). Green points indicate the actual limits on the candidate sources. The green line indicates the sensitivity for the combined search. Blue and red curves/points indicate the sensitivities for the individual IceCube and ANTARES analyses, respectively. 
the Southern sky search has a post trial p-value of 0.24 (significance of $0.7 \sigma$ ). In the source list search the candidate with the highest significance corresponds to HESS J1741-302, with a post-trial p-value of 0.11 (significance of $1.2 \sigma$ ). Both of the results are compatible with the background-only hypothesis and no significant excess is found. Flux upper limits for each of the source candidates have been calculated for $E^{-2}$ and $E^{-2.5}$ power-law energy spectra, as well as for $E^{-2}$ spectra with cut-offs at energies of $1 \mathrm{PeV}, 300 \mathrm{TeV}$, and $100 \mathrm{TeV}$. Because of their complementary nature, with IceCube providing more sensitivity at higher energies and ANTARES at lower energies, a joint analysis of future data sets will continue to provide the best point-source sensitivity in critical overlap regions in the Southern sky, where neutrino emission from Galactic sources in particular may be found.

\section{Acknowledgments}

The authors acknowledge the support of the Spanish MINECO through project FPA201237528-C02-01, the MultiDark Consolider Project CSD2009-00064, the Generalitat Valenciana via Prometeo-II/2014/079, and of Universitat de València, Atracció de Talent.

\section{References}

[1] Achterberg, A., Ackermann, M., Adams, J., et al. (IceCube Collaboration) 2006, APh, 26, 155.

[2] Ageron M., Aguilar J. A., Samarai I. Al et al. (ANTARES Collaboration) 2011, Nucl. Instrum. Meth., A $656,11$.

[3] Aartsen, M. G., et al. (IceCube Collaboration) 2013c, Sci, 342, 1242856.

[4] Aartsen, M. G., et al. (IceCube Collaboration) 2013b, Phys. Rev. Lett.,113, 101101.

[5] Adrián-Martínez S., Samarai I.Al, Albert A. et al. (ANTARES Collaboration) 2012a, Phys. Lett. B, $714,224$.

[6] Adrián-Martínez S., Samarai I.Al, Albert A. et al. (ANTARES Collaboration) 2013b, Eur. Phys. J. C, $73,2606$.

[7] Aartsen M. G., Abbasi R., Abdou Y. et al. (IceCube Collaboration) 2013d, ApJ, 779, 132.

[8] Adrián-Martínez S., Albert A., André M., et al. (ANTARES Collaboration) 2014, ApJ, 786, L5.

[9] Krymskii G.F., 1997, Soviet Physics Doklady, 22, 327.

[10] Blandford, R.D. \& Ostriker, J.P, 1978, ApJ, 221, L29.

[11] Aartsen M. G., Ackermann M., Adams J. et al. (IceCube Collaboration), 2015, Phys. Rev. D, 91, 022001.

[12] Kappes A., Hinton J., Stegmann C. \& Aharonian F. A., 2007, ApJ, 656, 870.

[13] Adrián-Martínez S., Samarai I. Al., Albert A. et al. (ANTARES Collaboration), 2012, ApJ, 760, 53

[14] Abbasi R., Abdou Y., Abu-Zayyad T. et al. (IceCube Collaboration), 2011, ApJ, 732, 18 